\title{
Development of Driver-Behavior Model Based on WOA-RBM Deep Learning Network
}

\author{
Junhui Liu $\left(\mathbb{D},{ }^{1,2}\right.$ Yajuan Jia, ${ }^{2}$ and Yaya Wang ${ }^{2}$ \\ ${ }^{1}$ The School of Electro-Mechanical Engineering, Xidian University, \\ The Key Laboratory of Electronic Equipment and Structure Design (Xidian University), Ministry of Education, \\ Xi'an 710071, China \\ ${ }^{2}$ The School of Electro Engineering, Xi'an Traffic Engineering Institute, Xi'an 710300, China \\ Correspondence should be addressed to Junhui Liu; liujunhui@stu.xidian.edu.cn
}

Received 2 August 2020; Revised 2 September 2020; Accepted 15 September 2020; Published 29 September 2020

Academic Editor: Petr Dolezel

Copyright (c) 2020 Junhui Liu et al. This is an open access article distributed under the Creative Commons Attribution License, which permits unrestricted use, distribution, and reproduction in any medium, provided the original work is properly cited.

\begin{abstract}
Human drivers' behavior, which is very difficult to model, is a very complicated stochastic system. To characterize a high-accuracy driver behavior model under different roadway geometries, the paper proposes a new algorithm of driver behavior model based on the whale optimization algorithm-restricted Boltzmann machine (WOA-RBM) method. This method establishes an objective optimization function first, which contains the training of RBM deep learning network based on the real driver behavior data. Second, the optimal training parameters of the restricted Boltzmann machine (RBM) can be obtained through the whale optimization algorithm. Finally, the well-trained model can be used to represent the human drivers' operation effectively. The MATLAB simulation results showed that the driver model can achieve an accuracy of $90 \%$.
\end{abstract}

\section{Introduction}

Driver models can be applied to (1) vehicle dynamics [1] including vehicle component design, vehicle dynamics analysis, overall vehicle stability analysis, and design of onboard controls; (2) intelligent transport systems (ITS) $[2,3]$ including simulation of traffic flow based on the control theory models of driver behavior and modeling drivers' risk taking behavior; (3) driverless vehicle systems [4]; and (4) traffic energy consumption systems [5]. Traffic energy consumption systems are different from the vehicle dynamics simulation. The traffic energy consumption system will be affected by the road, so our research focuses on how the road grade impacts on the driver's behavior characteristics. Driving behaviors, including acceleration behavior, deceleration behavior, and uniform behavior, have impacts on the driving safety $[6,7]$, vehicle fuel consumption [8-10], and air pollution [11]. Designing a drive cycle of the vehicle requires investigating and collecting the practical driving data, analyzing the experimental data, and establishing the road vehicle driving conditions using relevant mathematical theoretical methods. The vehicle speed of this paper is collected based on the distance, and we also considered how the road grade influences driving speeds in its operating conditions. The operating conditions of the resulting vehicles can be used to determine the vehicle's fuel consumption and the technical development as well as evaluation of new models. It is very important to establish an accurate driver behavior model. The main factors that affect the accuracy of the driver behavior model include road geometry and weather condition $[12,13]$. Since the driver behavior is a very complicated stochastic system [14-17], designing driver behavior modeling is a very challenging task.

At present, the main achievements of research on driver behavior modeling technology are as follows. Cai et al. [18] developed a new concept of the driving fingerprint map to represent driving characteristics. Miyajima and Takeda [19] proposed a driver behavior modeling method by using on-road driving data. The method is realized through statistical machine-learning techniques, such as hidden Markov models and deep learning. Angkititrakul et al. [20] proposed a stochastic 
driver behavior model based on Gaussian mixture model framework. This proposed method allows adaptation scheme to enhance the model capability to better represent particular driving characteristics of interest. Shi et al. [21] proposed to evaluate driving styles by normalizing driving behavior based on personalized driver modeling. An aggressiveness index is proposed to quantitatively evaluate driving styles in this method, which can be applied to detect abnormal driving behavior. Yamada and Takahashi [22] proposed a driver behavior modeling method based on real traffic data under varying environmental conditions. In this method, the driving speed is assumed to be a function of several factors such as overall traveling schedule, speed, and road surface conditions. Taniguchi et al. [23] proposed an unsupervised learning method, which is established on the basis of the original double articulation analyzer model. This method predicts possible scenarios of driving behavior by segmenting and modeling incoming driving behavior time series data. Okuda et al. [24] proposed a probability-weighted autoregressive exogenous model wherein the multiple autoregressive exogenous models are composed of the probabilistic weighting functions. This model can represent the actual driving behavior. There are plentiful publications on this topic using different optimization approaches, e.g., the instantaneous optimization algorithm [25], wolf pack algorithm [26], and genetic algorithm [27, 28].

However, methods in $[19,20]$ are very complex and strongly depend on historical data. Shi et al. [21], propose to quantitatively evaluate driving styles by normalizing driving behavior based on personalized driver modeling. The results show that the prediction accuracy of driving behavior modeling will be affected by complex environment. The establishment of methods in [22-24, 29] requires a large amount of actual driving data as measurement data, which is also strongly dependent on historical data.

To solve these problems, the paper presents a new method of driver behavior model based on WOA-RBM. This method establishes an objective function, which contains the training process of RBM based on real driver behavior data. Then, the best training parameters of RBM are obtained through WOA. Finally, the RBM after training based on the best training parameters can be used to build the driver behavior model.

This paper is organized as follows. Section 2 describes the driving data collection. Section 3 presents the process of driving behavior modeling based on WOA-RBM. Section 4 shows the experimental results, which prove that the proposed method in this paper can achieve a better performance. Conclusions are offered in Section 5.

\section{Driving Data Collection}

In this section, the driving data come from the measurement data along the highway 120 near Manteca, CA, USA. In order to build a highly accurate driver behavior model, more than 2000 different drivers' driving behavior data were collected for each route. The measurement data were collected in Manteca during June 17th-July 28th (six weeks), 2018 [30]. Vehicle speeds are measured at 9 points identified in Figure 1. From the map (Figure 1), this highway is an approximately straight road. When modeling the driver, only the influence of the road grade on the speed of the driver is considered, so the curvature of the road is not displayed. In future research, we will take curvature of the road into building the driver model. The road grade is small, but it still has a great impact on the energy consumption of the vehicle. The energy reduction comes from two reasons. First, if a vehicle has constant speed or accelerates, less energy is consumed by the power plant (the engine and/or the electric motor) to drive the vehicle downhill than on a flat road because the gravity contributes positive work to overcome the negative work by aerodynamic drag and tire rolling resistance. The vehicle's potential energy is partly converted into its kinetic energy. Second, if a vehicle decelerates on a flat road or a downhill, the reduced kinetic energy is wasted by the brake as heat. On the other hand, if the vehicle decelerates on an uphill, part of the decreased kinetic energy is converted into the vehicle's potential energy and less kinetic energy is wasted by the brake. The gained potential energy can later be converted back into kinetic energy during a downhill. In summary, road slope change turns the vehicle's potential energy into an energy buffer to store the kinetic energy. The data are collected in 5-minute interval between 00:00 and 23:55 every day at each measurement point. Vehicle speeds at positions other than the 9 points are estimated by linear interpolation. The studied route stretches along $6.1 \mathrm{~km}$ of highway driving. The altitude varies from approximately 9 to $17 \mathrm{~m}$. And the origin altitude is approximately $13 \mathrm{~m}$ while the terminal altitude is approximately $17 \mathrm{~m}$. The road is sampled by 305 even steps with the step length $\Delta s=6100 / 305=20 \mathrm{~m}$. The recorded speed trajectories for the first week and the altitude of the road are shown in Figures 2 and 3. The drivers generally increase the velocity between 4 and $6 \mathrm{~km}$ because this section of the road sets speed limits.

Figure 4 shows the slope information of road environment. When the slope is larger than 0 , the road is an uphill road; when the slope is lower than 0 , the road is a downhill road; when the slope is equal to 0 , the road is a flat road.

The driving data are shown in Table 1.

The data size is $2004 \times 306$, the data in each row represent the driving behaviors of 2004 different drivers in the same road section, and the data in each column show the driving behaviors of each driver at different sample points.

\section{Driver Behavior Model Based on WOA-RBM}

In this paper, a new driver behavior model based on WOA$\mathrm{RBM}$ is proposed, which imitates human driving behavior during real-world driving. This new method is designed based upon the theory of RBM and WOA.

3.1. Deep Learning Network Based on RBM. The deep learning network based on RBM can solve the problem of the multilayer network training, which is also easy to realize $[31,32]$. The structure of deep learning neural network based on RBM is shown in Figure 5. 


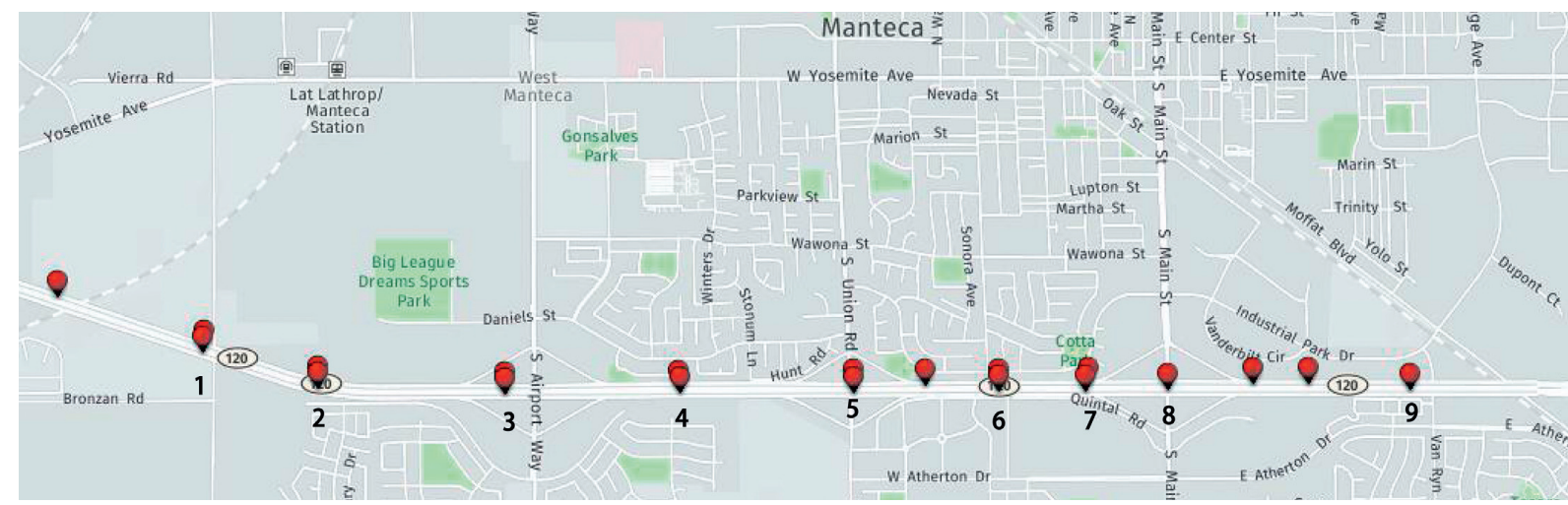

FIGURE 1: Route of driving data collection.

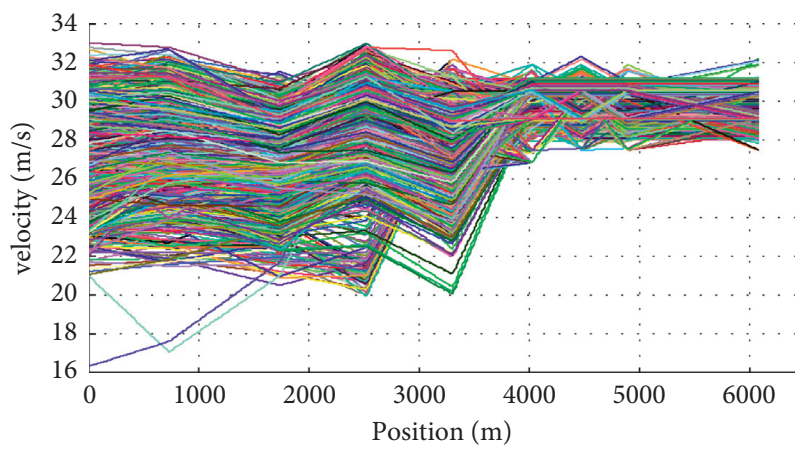

FIgURE 2: The data of different human driver operations.

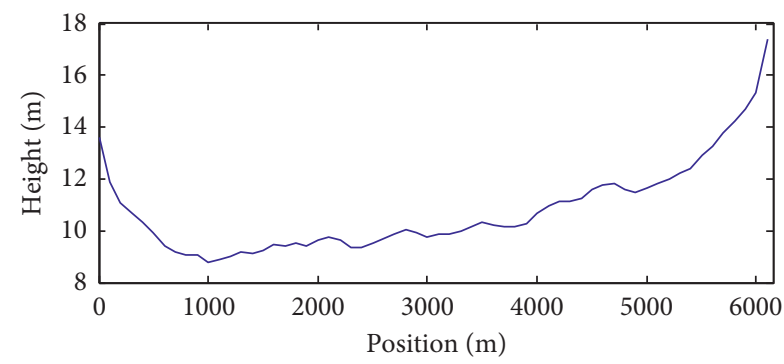

Figure 3: The altitude profile of the road.

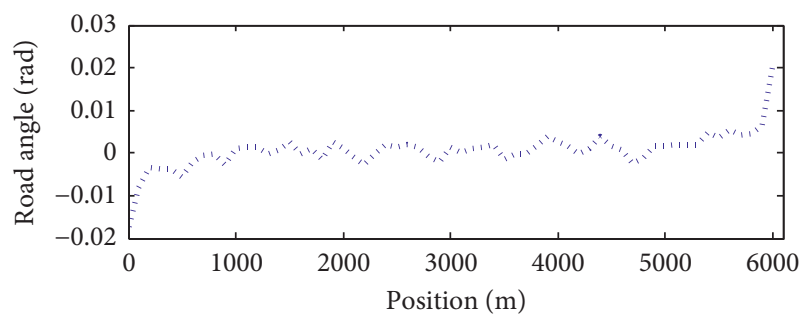

Figure 4: Slope information of road environment.

There are two RBMs and one backpropagation (BP) network in this structure, with a hidden layer and a visible layer in each RBM, both of which are connected through a two-way connection between layers, as shown in Figure 6.

As in references [31,32], the equation of RBM can be defined as follows:

$$
E(v, h)=-\sum_{i \in v} a_{i} v_{i}-\sum_{i \in h} b_{i} h_{i}-\sum_{i, j} v_{i} h_{i} W_{i j}
$$

where $E(v, h)$ is the energy function between the input $v$ vector and the hidden layer output vector $h, W_{i j}$ is a connection weight matrix, $v_{i}$ is the visible layer, $h_{i}$ is the hidden layer, $a_{i}$ is the bias of visible node $i$, and $b_{j}$ is the bias of hidden node $j$.

The probability of each visible $v$ and hidden layer $h$ can be defined as

$$
p(v, h)=\frac{1}{\sum_{v, h} e^{-E(v, h)}} e^{-E(v, h)} .
$$

The logarithmic gradient of weight in (2) can be calculated as

$$
\frac{\partial \log p(v, h)}{\partial W_{i j}}=\left\langle v_{i} h_{j}\right\rangle_{\mathrm{data}}-\left\langle v_{i} h_{j}\right\rangle_{\mathrm{model}}
$$

where $\left\langle v_{i} h_{j}\right\rangle_{\text {data }}$ is the mean value of data and $\left\langle v_{i} h_{j}\right\rangle_{\text {model }}$ is the mean value of model. Therefore, the learning rules of RBM can be computed as

$$
\Delta W_{i j}=\operatorname{Lr} \times\left(\left\langle v_{i} h_{j}\right\rangle_{\text {data }}-\left\langle v_{i} h_{j}\right\rangle_{\text {model }}\right),
$$

where $L r$ is the learning rate of RBM.

Similarly, the parameter of indexes in equation (1) can be calculated as follows:

$$
\begin{gathered}
\Delta a_{i}=\operatorname{Lr} \times\left(\left\langle v_{i}\right\rangle_{\mathrm{data}}-\sum_{k=1}^{N}\left\langle v_{i}\right\rangle_{k}\right), \\
\Delta b_{j}=\operatorname{Lr} \times\left(\left\langle h_{j}\right\rangle_{\mathrm{data}}-\sum_{k=1}^{N}\left\langle h_{j}\right\rangle_{k}\right) .
\end{gathered}
$$

From the above analysis, it is noted that the parameters affecting RBM training performance include the initial value of $\left\{\mathrm{Lr}, a_{i}, b_{j}, W_{i j}\right\}$, the number of hidden layer $h$, and the number of visible layer $v$. Therefore, it is significant to choose the appropriate parameters.

According to the above principle, we assume that the training process of RBM is represented as follows: 
TABLE 1: Segments of the vehicle speeds.

\begin{tabular}{lcccccccc}
\hline \multirow{2}{*}{ Different drivers } & \multicolumn{9}{c}{ Speeds at sample points $(\mathrm{m} / \mathrm{s})$} \\
& 1 & 2 & 3 & 4 & 5 & 6 & 7 \\
\hline 1 & 31.2900 & 31.2851 & 31.2803 & 31.2754 & 31.2706 & 31.2657 & 31.2608 & 31.2560 \\
2 & 30.3960 & 30.3960 & 30.3960 & 30.3960 & 30.3960 & 30.3960 & 30.3960 & 30.3960 \\
3 & 30.5301 & 30.5252 & 30.5204 & 30.5155 & 30.5107 & 30.5058 & 30.5009 & 30.4961 \\
4 & 30.8877 & 30.8804 & 30.8731 & 30.8658 & 30.8585 & 30.8531 & 30.8440 & 30.8367 \\
5 & 30.5301 & 30.5398 & 30.5495 & 30.5593 & 30.5690 & 30.5787 & 30.5884 & 30.5981 \\
\hline
\end{tabular}

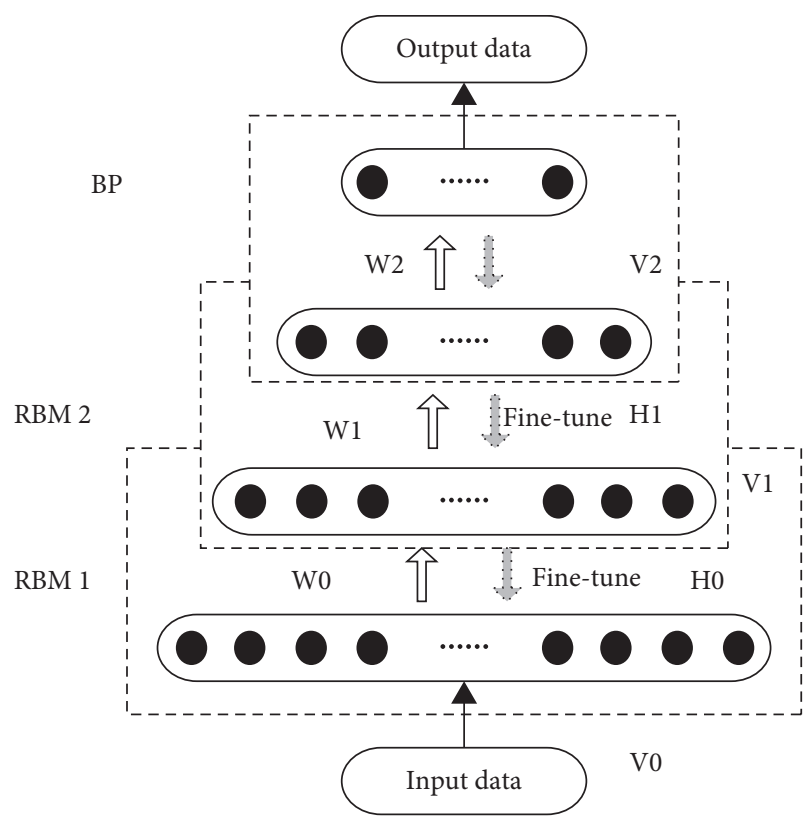

FIGURE 5: The structure of deep learning neural network based on RBM.

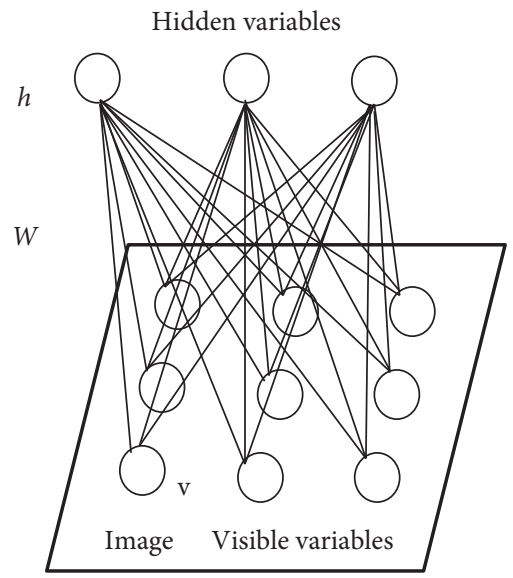

FIgURE 6: The basic structure of RBM.

$$
\operatorname{err}=f_{\mathrm{RBM}}\left(\operatorname{Lr}, v, h, a_{i}, b_{j}, W_{i j}\right)
$$

where err represents the training error. The smaller the training error is, the closer the driving behavior model similar to the actual driving behavior is.
3.2. Calculation of Optimal RBM Training Parameters. Obviously, the calculation of optimal RBM training parameters is an NP-hard problem. It difficult to calculate the global optimal value directly. To resolve this problem, the paper proposes the whale optimization algorithm (WOA) [33-35]. The main steps of WOA are as follows:

Step 1. Walking and foraging stage: the humpback whale can find the position of food, and the behavior of humpback whale is defined as

$$
\vec{D}=\left|\vec{C} \cdot \vec{X}_{\text {rand }}-\vec{X}_{t}\right|
$$

where $\vec{C}$ is a coefficient vector, $\vec{D}$ is the distance between the whales and food, $\vec{X}_{r \text { and }}$ is the random position, and $\vec{X}_{t}$ is the current position.

The position of the next moment can be defined as

$$
\vec{X}_{t+1}=\vec{X}_{r \text { and }}-\vec{A} \times \vec{D}
$$

The vectors $\vec{A}$ and $\vec{C}$ in equations (7) and (8) can be presented as

$$
\begin{aligned}
& \vec{A}=2 \vec{a} \cdot \vec{r}-\vec{a}, \\
& \vec{C}=2 \cdot \vec{r},
\end{aligned}
$$

where $\vec{r}$ is a random vector whose values lie in the range of $[0,1]$ and $\vec{a}$ is the value which is decreased linearly from 2 to 0 .

In this paper, the whale population $\vec{X}_{t}$ is presented as

$$
\vec{X}_{t}=\left[\operatorname{Lr}, v, h, a_{i}, b_{j}, W_{i j}\right]
$$

Step 2. Encircling and contracting stage: when the humpback whale finds the target food, the other whales will go to the position of the humpback whale and surround the food. The equation of this stage can be presented as follows: 


$$
\begin{aligned}
\vec{D} & =\left|\vec{C} \cdot \vec{X}_{t}^{*}-\vec{X}_{t}\right|, \\
\vec{X}_{t+1} & =\left|\vec{X}_{t}^{*}-\vec{A} \cdot \vec{D}\right|,
\end{aligned}
$$

where $\vec{X}_{t}^{*}$ is the vector of random position, which is chose to the current whale population.

Step 3. Spiral predation stage: all the whales will move in a spiral direction of the optimal position of the humpback whale, and then the whales will generate many bubbles to surround the food for predation; the equation of this stage is

$$
\vec{X}_{t+1}=\vec{X}_{t}^{*}+\vec{D}^{\prime} \cdot e^{b \vec{l}} \cdot \cos (2 \pi \vec{l}),
$$

where $\vec{D}^{\prime}=\left|\vec{X}_{t}^{*}-\vec{X}_{t}\right|$ is the distance between the whale and the optimal solutions, $b$ is a shape of logarithmic spiral, and $l$ is a random number in the range of $[-1,1]$.

Finally, we can define a random value $p$ to distinguish the contraction-bounding stage from the spiral predator. The equation is as follows:

$$
\vec{X}_{t+1}= \begin{cases}\vec{X}_{t}^{*}+\vec{D} \cdot e^{b \vec{l}} \cdot \cos (2 \pi \vec{l}), & p \geq 0.5 \\ \vec{X}^{*}-\vec{A} \cdot \vec{D}, & p<0.5\end{cases}
$$

To sum up, the flowchart of WOA is shown in Figure 7.

3.3. Driver Behavior Model Based on WOA-RBM. Upon the completion of the optimization process, we obtain the optimal parameters:

$$
\overrightarrow{X_{\mathrm{opt}}}=\left[\operatorname{Lr}_{\mathrm{opt}}, v_{\mathrm{opt}}, h_{\mathrm{opt}}, a_{i, \mathrm{opt}}, b_{j, \mathrm{opt}}, W_{i j, \mathrm{opt}}\right] \text {. }
$$

Subsequently, the optimized RBM model can be expressed as

$$
E(v, h)=-\sum_{i \in v_{\mathrm{opt}}} a_{i, \mathrm{opt}} v_{i, \mathrm{opt}}-\sum_{i \in h_{\mathrm{opt}}} h_{i, \mathrm{opt}} h_{i, \mathrm{opt}}-\sum_{i, j} v_{i, \mathrm{opt}} h_{i, \mathrm{opt}} W_{i j, \mathrm{opt}} .
$$

The learning rules of optimized RBM can be calculated as

$$
\begin{gathered}
\Delta W_{i j}=\operatorname{Lr}_{\mathrm{opt}} \times\left(\left\langle v_{i, \mathrm{opt}} h_{j, \mathrm{opt}}\right\rangle_{\mathrm{data}}-\left\langle v_{i, \mathrm{opt}} h_{j, \mathrm{opt}}\right\rangle_{\mathrm{model}}\right), \\
\Delta a_{i}=\operatorname{Lr}_{\mathrm{opt}} \times\left(\left\langle v_{i, \mathrm{opt}}\right\rangle_{\mathrm{data}}-\sum_{k=1}^{N}\left\langle v_{i, \mathrm{opt}}\right\rangle_{k}\right) \\
\Delta b_{j}=\operatorname{Lr}_{\mathrm{opt}} \times\left(\left\langle h_{j, \mathrm{opt}}\right\rangle_{\mathrm{data}}-\sum_{k=1}^{N}\left\langle h_{j, \mathrm{opt}}\right\rangle_{k}\right) .
\end{gathered}
$$

According to the theories introduced above, the driver behavior model based on WOA-RBM is shown in Figure 8. The driver behavior model and the vehicle model constitute a closedloop control system. The driver behavior model is actually an inverse model of the controlled object (vehicle). In this paper, based on road test data, the learning control method is used to build a driver model through machine learning. Assume that the vehicle speed at a certain position is $V(s)$, and the driver presses

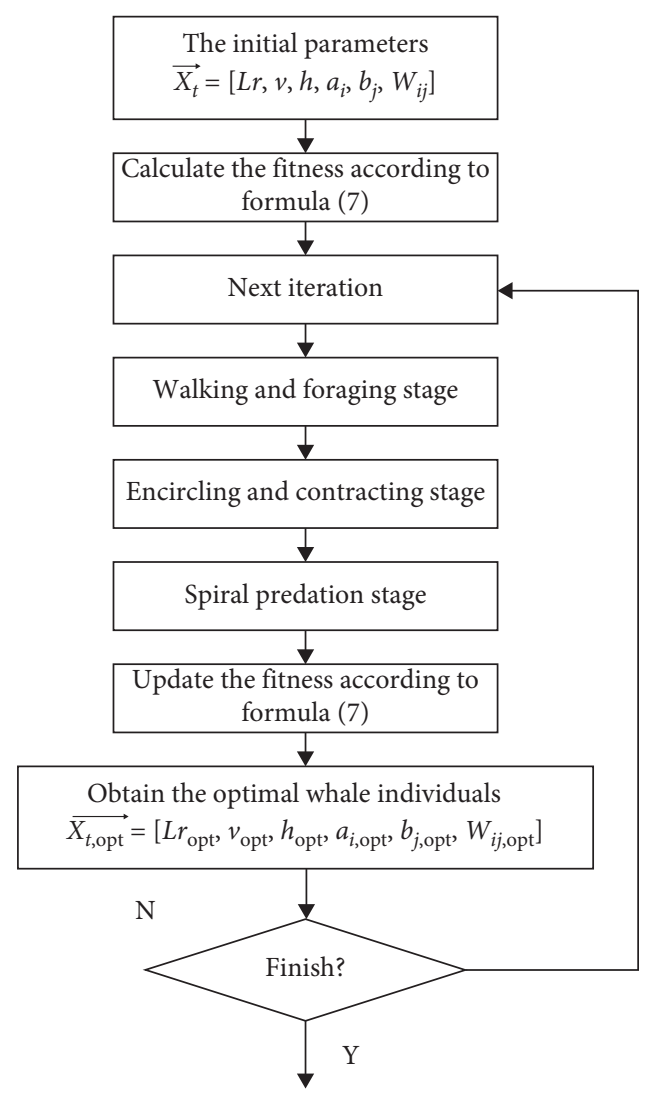

Figure 7: The flowchart of WOA.

down the accelerator pedal or brake pedal which as a consequence will affect the vehicle speed at the next position $V(s+1)$. The expected speed of the next position is $V_{e}(s+1)$, i.e., the road's speed limit. The collected vehicle speed data are used to train the optimal control parameters which are input into the driver behavior model. At the same time, the road grade, the actual speed of the vehicle, and the deviation between the actual speed and the expected speed are also input into the driver behavior model. Subsequently, the driver behavior model outputs acceleration or deceleration to control the driving of the vehicle.

Figure 8 shows the proposed driver behavior model. In this model, the sampled driver data are used to train the WOA-RBM model. The optimal parameters $\mathrm{Lr}_{\mathrm{opt}}, v_{\mathrm{opt}}, h_{\mathrm{opt}}, a_{i, \mathrm{opt}}, b_{j, \mathrm{opt}}$, and $W_{i j \text { opt }}$ of deep learning network are obtained after WOA optimization. The WOA-RBM model after training with the optimal training parameters is the driving behavior model. Finally, the driving behavior model is used in a vehicle control system. In Figure 8, the "road information" specifically refers to the road grade. $\Delta V$ indicates the difference between actual speed and desired speed. The whole closed-loop control system output is the vehicle speed. Drive operation is acceleration or deceleration.

\section{Experiment}

The proposed driving behavior model in this paper is simulated and validated through MATLAB 2017b platform. The performance of the proposed driving behavior model 


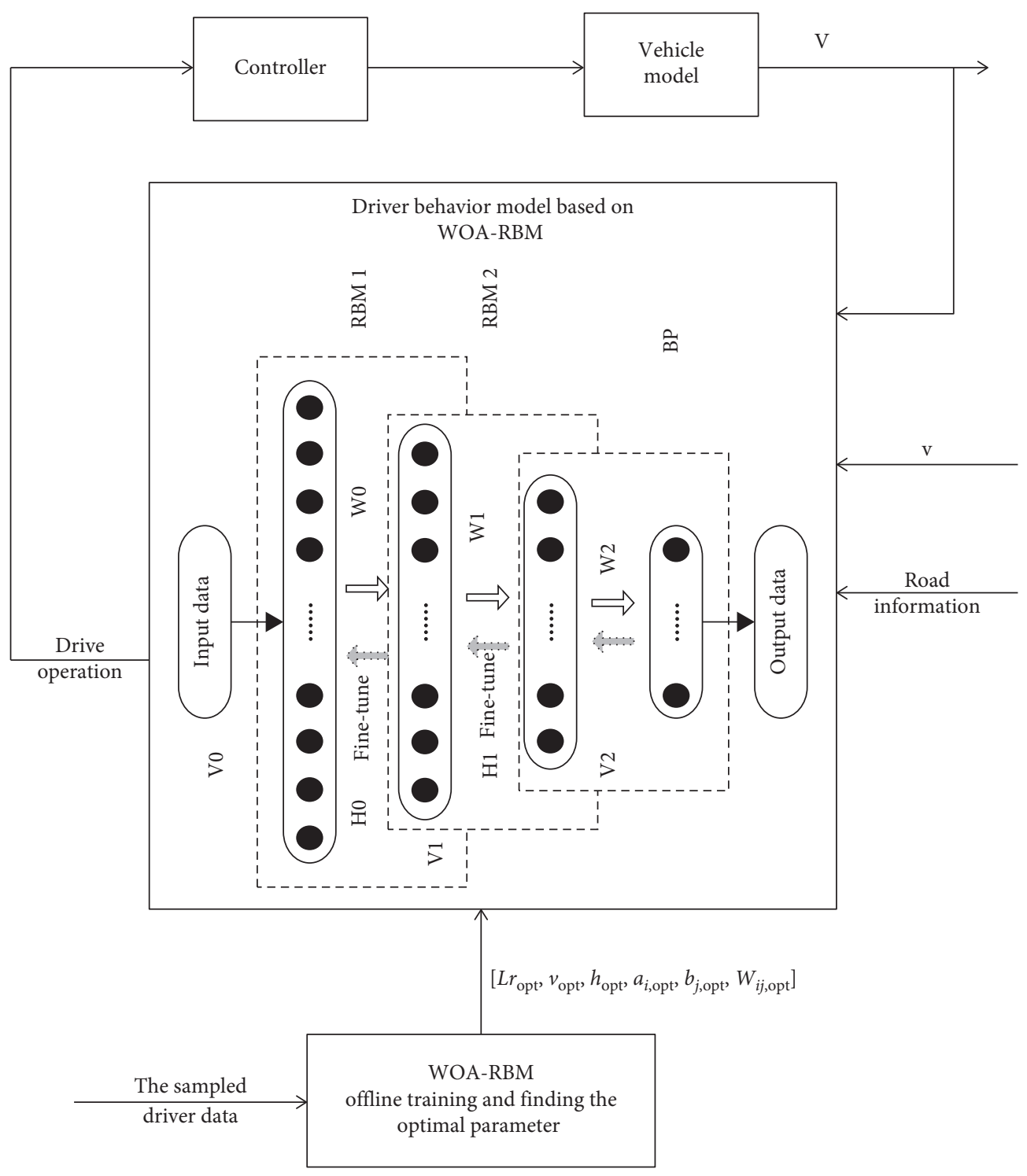

Figure 8: The structure of driver behavior model based on WOA-RBM.

and several other existing driving models are also compared. The configuration of the computer is as follows: Intel i- $9700 \mathrm{~K}$ processor with 8 cores, CPU frequency of $4 \mathrm{GHz}$, memory size of $16 \mathrm{G}, 64$ bit Windows 7 Professional operation system, and Nvidia GeForce GTX 980 graphics card.

4.1. The Parameters. The initial parameters of the proposed driver behavior model are shown in Table 2.

In this experiment, 100 different road sections were collected, and about 2000 drivers' driving data were collected for each route. The driving behavior data of each driver in each route were collected at 306 points.

In this paper, 70 groups are randomly selected as training samples, and the remaining 30 groups are selected as testing samples.

4.2. WOA Optimization Process. The optimization process of WOA is illustrated in Figure 9.
TABLE 2: The initial parameters.

\begin{tabular}{lcc}
\hline No. & Variable & Value \\
\hline 1 & Number of whale population & 50 \\
2 & Iteration times & 100 \\
3 & WOA search space dimension & 10 \\
4 & Initial learning rate & 0.1 \\
5 & Batch size & 10 \\
6 & Number of hidden layers & 4 \\
7 & Number of visible layers & 2 \\
8 & Training times & 50 \\
9 & Number of driver behavior data & 2000 \\
10 & Length of each driver behavior data & 300 \\
11 & Average velocity & $30 \mathrm{~m} / \mathrm{s}$ \\
12 & Simulation platform & MATLAB 2017b \\
\hline
\end{tabular}

Figure 9 shows that when the number of iterations is 9, WOA minimizes the training error of RBM deep learning neural network. The comparison of RBM parameters before and after optimization is shown in Table 3. 


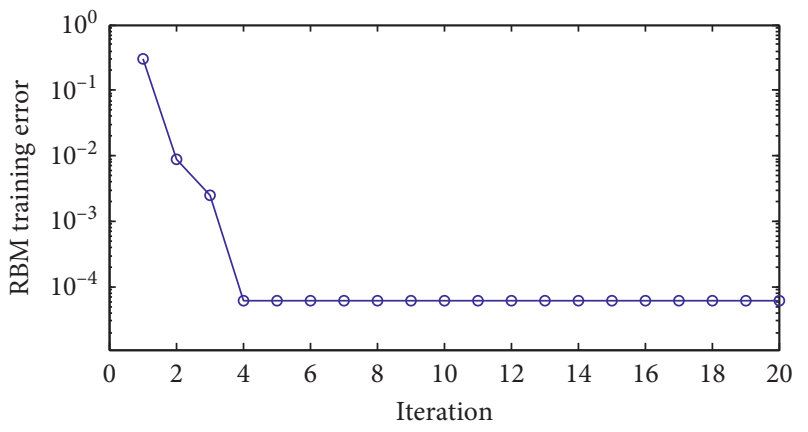

FIgURE 9: The optimization process of WOA.

TABle 3: Simulation parameters.

\begin{tabular}{lccc}
\hline No. & Parameter & Initial value & Optimal value \\
\hline 1 & $L r$ & 1 & 0.0039 \\
2 & $v$ & 1 & 2 \\
3 & $h$ & 1 & 2 \\
4 & $a$ & 0.9134 & 0.0888 \\
5 & $b$ & 0.6325 & 0.1748 \\
6 & $W$ & 0.0975 & 0.6883 \\
\hline
\end{tabular}

The simulation results show that with the increase of the number of iterations, the training error of RBM deep learning network gradually reduces. When the number of iterations is 4, WOA minimizes the training error of RBM deep learning neural network, which is within a precision of $10^{-4}$. The comparison of RBM parameters before and after optimization is shown in Table 3. After optimized by WOA, the RBM optimal learning rate $\mathrm{Lr}$ is 0.0039 , the number of visual layers $v$ is 2 , the number of hidden layers $h$ is 2 , the optimal values of parameters $a$ and $b$ are 0.1748 and 0.6883 , respectively, and the optimal initial average of the initial network weights $w$ is 0.088 . Under these parameters, the training effect of RBM can obtain the optimal result.

4.3. The Performance of Driver Behavior Model. The information of the road is already shown in Figure 4. The actual driving behavior and the driving behavior predicted by the driver behavior model are simulated in Figure 10.

Figure 10 shows that the six drivers' behaviors from the output of the WOA-RBM-based driver behavior model are consistent with the actual driving behavior. Therefore, the driving behavior model proposed in this paper exhibits high prediction accuracy.

Furthermore, the standard error of estimate (SEE) is used to evaluate the proposed models by more driving behaviors, which is defined as

$$
\operatorname{SEE}(i)=\sqrt{\frac{\sum_{n=1}^{N}\left(\mathrm{dat}_{\mathrm{actual}}(i, n)-\mathrm{dat}_{\mathrm{WOA}-\mathrm{RBM}}(i, n)\right)^{2}}{N}},
$$

where dat ${ }_{\text {actual }}(i, n)$ are the actual driver behavior data, i.e., the vehicle speeds from the $i-$ th driver at the $n-$ th sampled point, and dat $_{\mathrm{WOA}-\mathrm{RBM}}(i, s)$ are the predicted driver behavior data from the $i-$ th driver at the $n-$ th sampled point.

The simulation result of SEE is shown in Figure 11.

Figure 11 shows that the SEE of the driving data and the actual data is obtained by the WOA-RBM model. The simulation results show that the SEE of more than 2000 different drivers is lower than one. Therefore, the driver behavior model based on WOA-RBM can correctly simulate the behavior of the driver.

Through training the 100 driving operation data in different routes, a high level of prediction accuracy is obtained. The corresponding test structure is shown in Figure 12:

From the results of simulation, it can be seen that for 100 different routes, the driving behavior prediction algorithm proposed in this paper can be obtained with lower SEE. Compared with the simulation results in Figure 13, for different routes, the SEE of the driving behavior prediction algorithm proposed in this paper is always close to 0.2 . It shows that the accuracy of the algorithm is high.

Overfitting means the training error is very small, while the generalization error is very large. Because the model may be too complex, it "remembers" the training samples, but its generalization error is very high. In the algorithm proposed in this topic, the dropout mechanism is used to prevent overfitting.

4.4. Comparison. To evaluate the performance of the WOARBM-based driver behavior model, some other driver behavior models are presented for comparison and analysis, including the model based on database of personal mobility driving [36], stochastic driver pedal behavior model [16], driver behavior modeling using on-road driving data [19], and driver behavior modeling using hidden Markov model based on genetic algorithm [28]. The simulation results of the four methods are shown in Figures 14-16.

The driving behavior predicted by the driver behavior model is shown in Figure 14. The simulation result indicates that the driving data obtained by the proposed WOA-RBM model are closer to the actual driving data.

The SEE value of the outputs of different driving models and the real driving data is calculated according to equation (17). It can be seen that the performance of the WOA-RBMbased driving model proposed in this paper is better than 


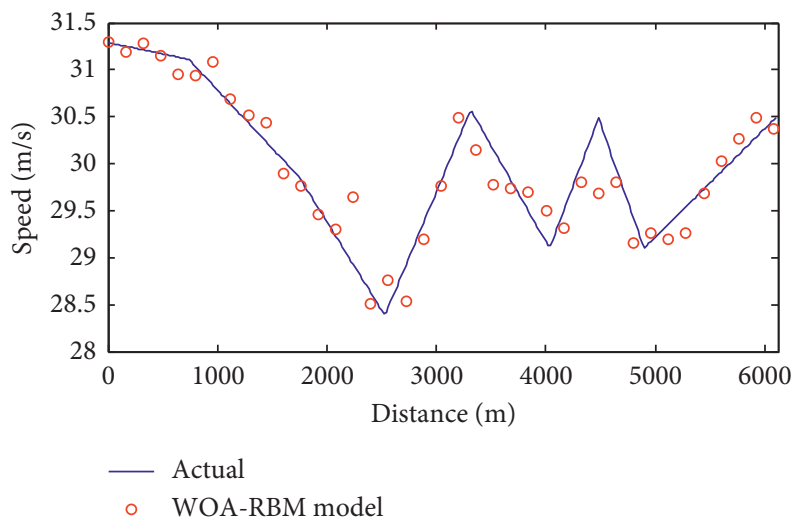

(a)

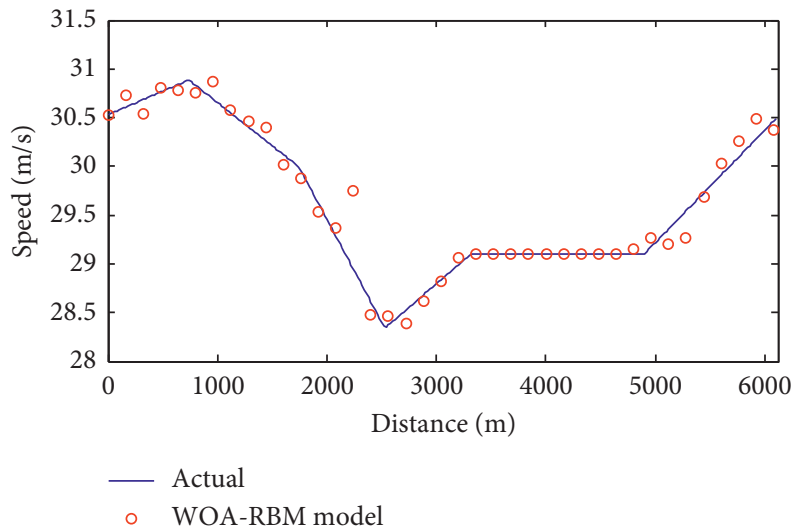

(c)

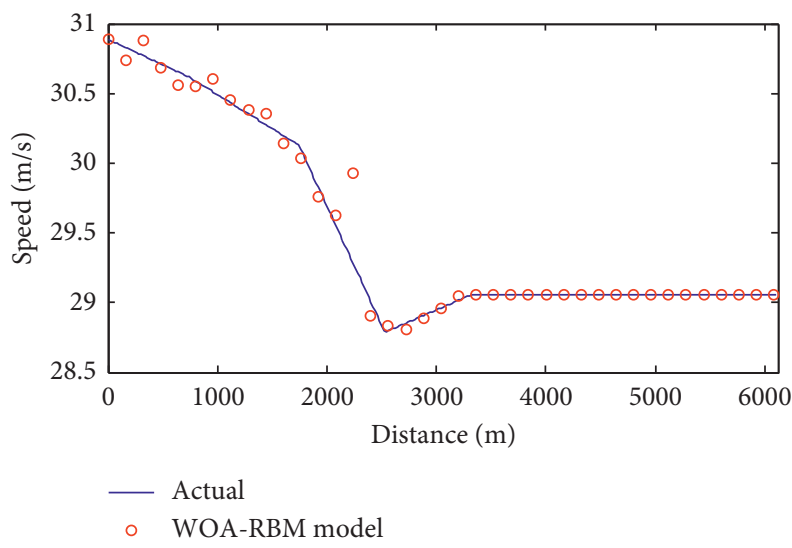

(e)

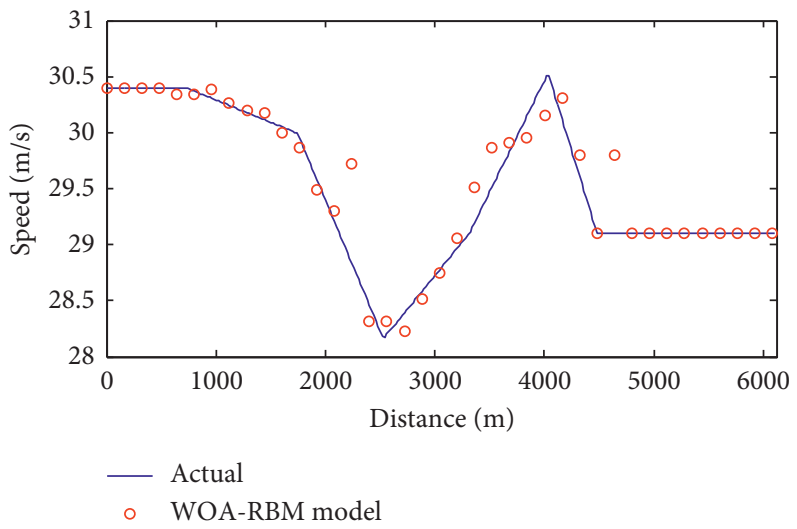

(b)

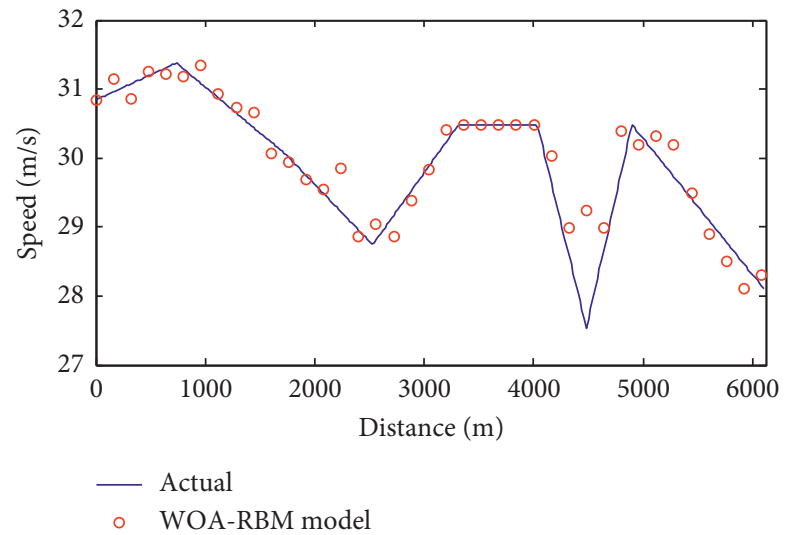

(d)

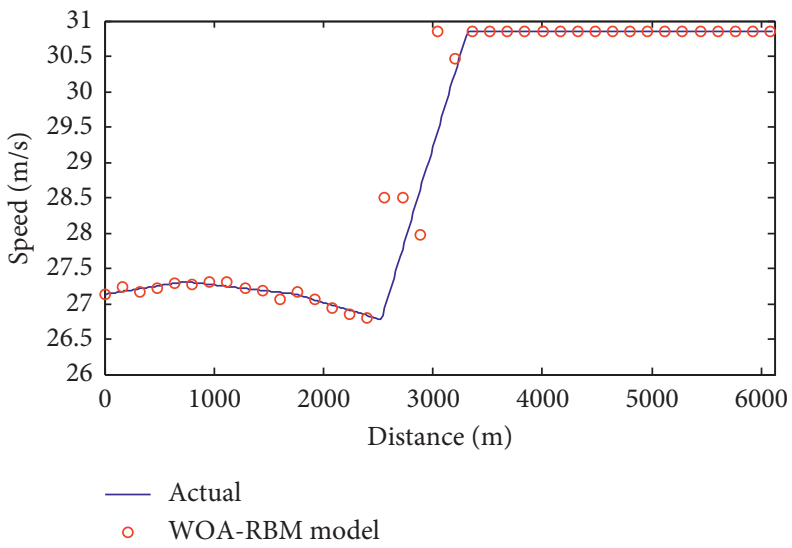

(f)

Figure 10: WOA-RBM-based driver behavior model prediction.

that of the driving models proposed in $[16,19,28,36]$. This is due to the fact that the WOA-RBM-based driving model proposed in this paper can obtain the optimal training parameters of RBM deep learning through WOA and can obtain a model more in line with actual driving data by learning driving training data.

Figure 13 shows that the SEE of the WOA-RBM model is the lowest, while the model in [28] is the largest.

Figure 13 shows the SEE of all the driver models. The driving data are added to verify the influence of different training data, which is shown in Table 4. Comparing the five driving models, the corresponding SEE values are calculated by equation (17) and the WOA-RBM driving behavior prediction model proposed in this paper has the highest prediction performance which has better performance than other algorithms with less training samples.

Table 5 shows the influence of different roads, including smooth road, less undulating road, and large undulating road. The simulation results show that the proposed WOARBM model has the best performance among all kinds of 


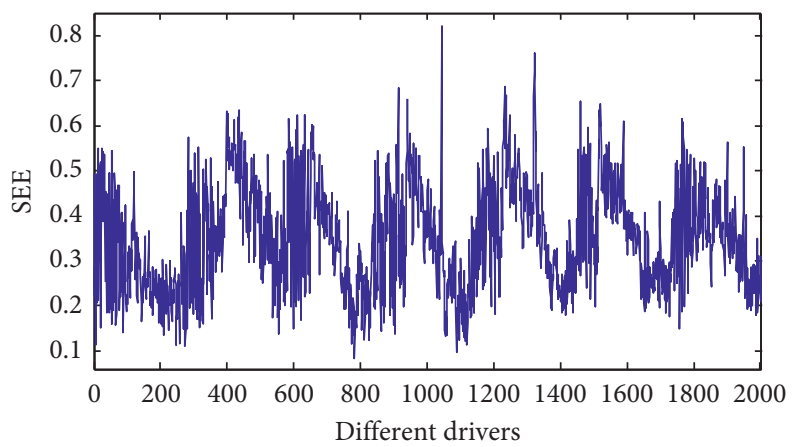

FIGURE 11: Simulation of SEE.

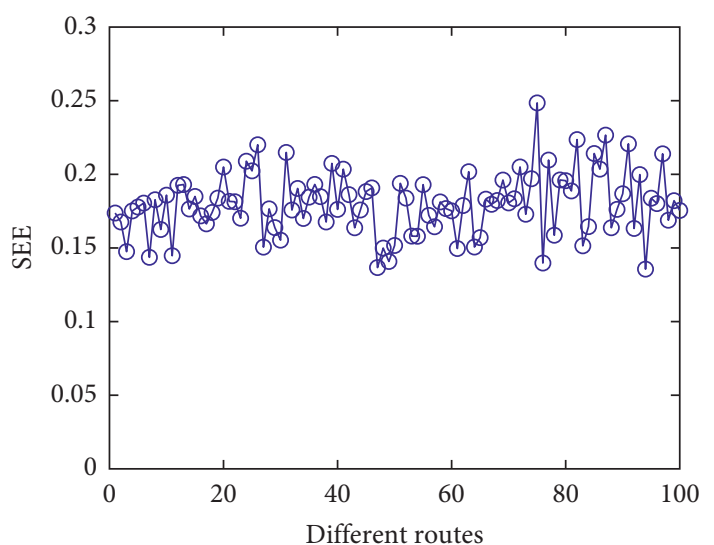

FIgURE 12: The different routes of SEE.

roads. Several remaining algorithms have larger prediction errors. Especially on road with large undulations, the difference becomes more and more obvious.

On the other hand, the correct and optimal driving behavior can also reduce the energy consumption of the vehicle. In our previous paper [29], the energy consumption model has been discussed. In this paper, the same energy consumption estimation method is used. The energy is calculated as the mechanical energy required for propelling the vehicle. The energy consumption of a vehicle can be estimated from its longitudinal dynamics, as illustrated in Figure 15. By Newton's law, we have the following equation:

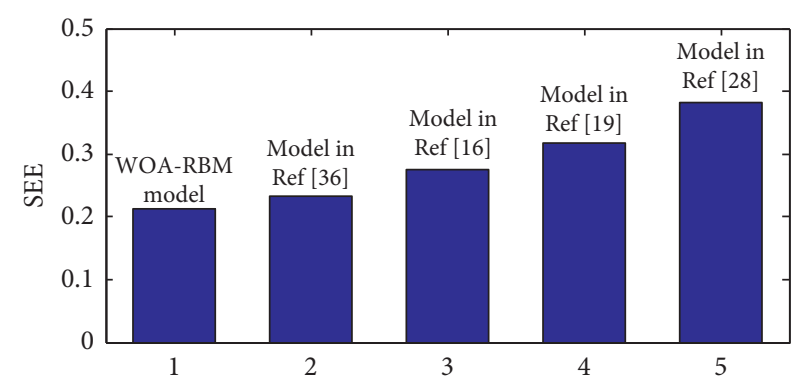

FIGURE 13: SEE of different driver behavior model predictions.

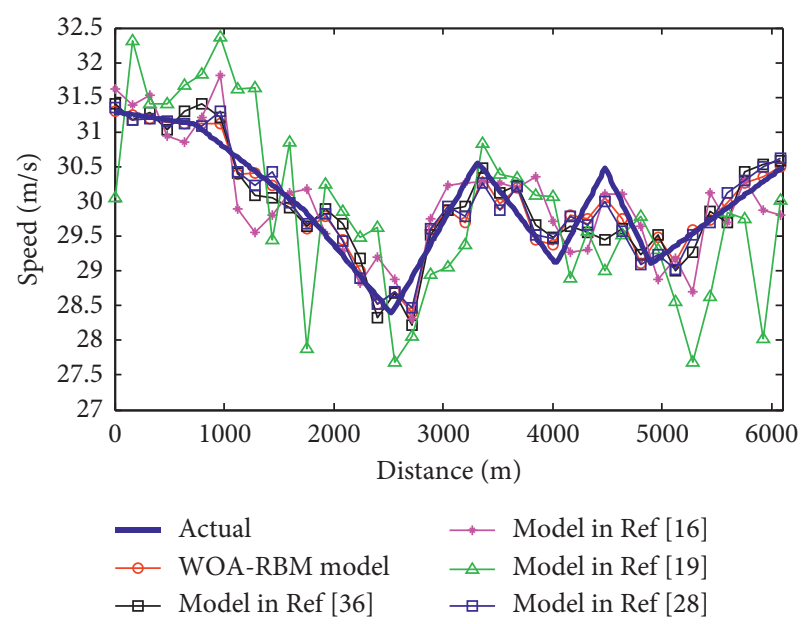

FIGURE 14: Different driver behavior model predictions.

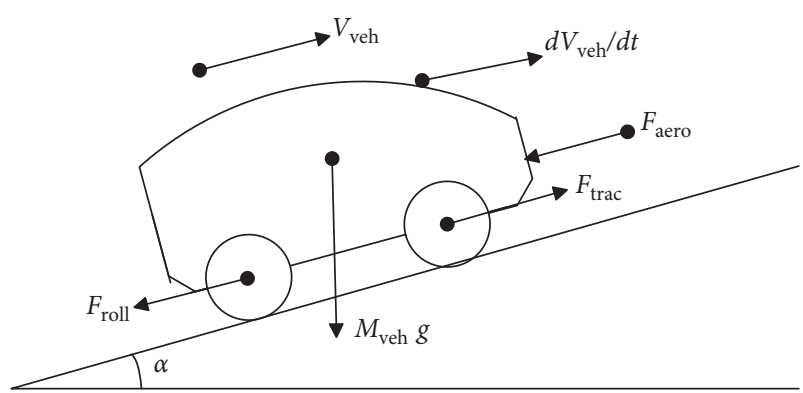

FIGURE 15: Longitudinal forces on a running vehicle.

$$
F_{\text {trac }}=M_{\mathrm{veh}} a+F_{\text {roll }}+F_{\text {aero }}+F_{\text {grade }}=M_{\text {veh }} a+c_{\text {roll }} M_{\text {veh }} g \cos \alpha+\frac{1}{2} \rho_{\text {air }} A_{f} c_{d} v_{\text {veh }}^{2}+M_{\text {veh }} g \sin \alpha,
$$

where $M_{\mathrm{veh}}$ is the vehicle mass, $a$ is the vehicle acceleration, $v_{\text {veh }}$ is the vehicle speed, $F_{\text {roll }}$ is the rolling friction, $F_{\text {aero }}$ is the aerodynamic drag, $F_{\text {grade }}$ is the force caused by the road slope, $F_{\text {trac }}$ is the traction force generated by the powertrain, $g$ is the gravity acceleration, $\alpha$ is the road grade angle in radiant, $c_{\text {roll }}$ is the rolling friction coefficient, $\rho_{\text {air }}$ is the density of the ambient air, $c_{d}$ is the air resistance coefficient, and $A_{f}$ is the frontal area of the vehicle. Figure 16 compares the performance of energy consumption.
The correct and optimal driving behavior can also reduce the energy consumption of vehicle. Figure 16 shows that the proposed WOA-RBM-based driver behavior model has lower energy consumption than other four models.

When operating a motor vehicle, providing the proper driving operation is essential, as it will reduce energy consumption. This means that frequent acceleration and braking is an improper driving method and will increase energy consumption. 


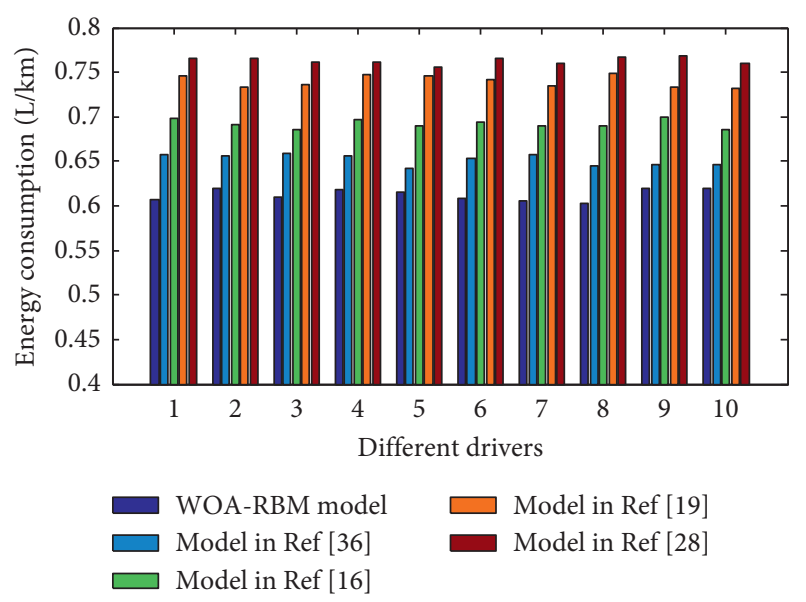

FIgURE 16: A comparison of energy consumption.

TABLe 4: The effect of the number of training samples on the performance of the algorithm.

\begin{tabular}{lccccc}
\hline $\begin{array}{l}\text { No. of training } \\
\text { samples }\end{array}$ & $\begin{array}{c}\text { SEE } \\
\text { RBM }\end{array}$ & $\begin{array}{c}\text { Ref } \\
{[36]}\end{array}$ & $\begin{array}{c}\text { Ref } \\
{[16]}\end{array}$ & $\begin{array}{c}\text { Ref } \\
{[19]}\end{array}$ & $\begin{array}{c}\text { Ref } \\
{[28]}\end{array}$ \\
\hline 200 & 0.205 & 0.22 & 0.252 & 0.311 & 0.358 \\
100 & 0.21 & 0.23 & 0.26 & 0.32 & 0.37 \\
80 & 0.225 & 0.264 & 0.32 & 0.354 & 0.412 \\
50 & 0.246 & 0.305 & 0.391 & 0.415 & 0.475 \\
20 & 0.289 & 0.372 & 0.445 & 0.482 & 0.523 \\
\hline
\end{tabular}

TABle 5: The effect of different roads on the performance of the algorithm.

\begin{tabular}{lcccccc}
\hline $\begin{array}{l}\text { Different } \\
\text { roads }\end{array}$ & $\begin{array}{c}\text { WOA- } \\
\text { RBM }\end{array}$ & CD & $\begin{array}{c}\text { Ref } \\
{[36]}\end{array}$ & $\begin{array}{c}\text { Ref } \\
{[16]}\end{array}$ & $\begin{array}{c}\text { Ref } \\
{[19]}\end{array}$ & $\begin{array}{c}\text { Ref } \\
{[28]}\end{array}$ \\
\hline $\begin{array}{l}\text { Smooth road } \\
\begin{array}{l}\text { Less } \\
\text { undulating }\end{array}\end{array}$ & 0.157 & 0.178 & 0.183 & 0.204 & 0.224 & 0.315 \\
$\begin{array}{l}\text { Large } \\
\text { undulating }\end{array}$ & 0.225 & 0.245 & 0.2753 & 0.283 & 0.348 & 0.410 \\
\hline
\end{tabular}

\section{Conclusion}

In this paper, a new algorithm of driver behavior model based on WOA-RBM deep learning network is proposed. The model establishes an objective function, which contains the training of deep learning network. The RBM after training based on optimal training parameters is used to predict the output action accurately through the road information. In the future, it is of great interest to collect huge amounts of real driving data from different roads and different drivers.

\section{Data Availability}

All raw data in this study are available free of charge. Readers who wish to repeat this study can do so through the following link: http://pems.dot.ca.gov/.

\section{Conflicts of Interest}

The authors declare that they have no conflicts of interest.

\section{Acknowledgments}

This study was supported by the Scientific Research Program funded by Shaanxi Provincial Education Department (Program no. 20JK0747).

\section{References}

[1] M. Plöchl and J. Edelmann, "Driver models in automobile dynamics application," Vehicle System Dynamics, vol. 45, no. 7-8, pp. 699-741, 2007.

[2] O. Derbel, T. Peter, H. Zebiri, B. Mourllion, and M. Basset, "Modified intelligent driver model for driver safety and traffic stability improvement," IFAC Proceedings Volumes, vol. 46, no. 21, pp. 744-749, 2013.

[3] S. Fernandez and T. Ito, "Driver behavior model based on ontology for intelligent transportation systems," in Proceedings of the 2015 IEEE 8th International Conference on ServiceOriented Computing and Applications (SOCA), pp. 227-231, Rome, Italy, October 2015.

[4] J. Meech and J. Parreira, "An interactive simulation model of human drivers to study autonomous haulage trucks," Procedia Computer Science, vol. 6, pp. 118-123, 2011.

[5] M. Zhang, S. Shi, W. Cheng, Y. Shen, and W. Cao, "Using the amce algorithm to high-efficiently develop vehicle driving cycles with road grade," IEEE Access, vol. 7, pp. 160449160458, 2019.

[6] C. Ma, W. Hao, W. Xiang, and W. Yan, "The impact of aggressive driving behavior on driver-injury severity at highway-rail grade crossings accidents," Journal of Advanced Transportation, vol. 2018, Article ID 9841498, 10 pages, 2018.

[7] Y. Ma, Z. Zhang, S. Chen, Y. Yu, and K. Tang, "A comparative study of aggressive driving behavior recognition algorithms based on vehicle motion data," IEEE Access, vol. 7, pp. 8028-8038, 2019.

[8] X. Liu, H. Xie, H. Ma, and S. Chen, "The effects of bus driver's behavior on fuel consumption and its evaluation indicator," Automotive Engineering, vol. 36, no. 11, pp. 1321-1326, 2014.

[9] D. Hari, C. J. Brace, C. Vagg, J. Poxon, and L. Ash, "Analysis of a driver behaviour improvement tool to reduce fuel consumption," in Proceedings of the 2012 International Conference on Connected Vehicles and Expo (ICCVE), pp. 208-213, 2012.

[10] F. Zheng, J. Li, H. J. van Zuylen, and C. Lu, "Influence of driver characteristics on emissions and fuel consumption," IET Intelligent Transport Systems, vol. 13, no. 12, pp. 17701779, 2019.

[11] D. W. Wyatt, H. Li, and J. E. Tate, "The impact of road grade on carbon dioxide $\left(\mathrm{CO}_{2}\right)$ emission of a passenger vehicle in real-world driving," Transportation Research Part D: Transport and Environment, vol. 32, pp. 160-170, 2014.

[12] J. Mclean, "Driver speed behaviour and rural road alignment design," Traffic Engineering \& Control, vol. 22, no. 4, pp. 208-211, 1981.

[13] S. H. Hamdar, L. Qin, and A. Talebpour, "Weather and road geometry impact on longitudinal driving behavior: exploratory analysis using an empirically supported acceleration modeling framework," Transportation Research Part C: Emerging Technologies, vol. 67, pp. 193-213, 2016. 
[14] K. Shaaban and H. Hamad, "Group gap acceptance: a new method to analyze driver behavior and estimate the critical gap at multilane roundabouts," Journal of Advanced Transportation, vol. 2018, no. 2, 9 pages, Article ID 1350679, 2018.

[15] A. M. Pérez-Zuriaga, F. J. Camacho-Torregrosa, A. García, and J. M. Campoy-Ungría, "Application of global positioning system and questionnaires data for the study of driver behaviour on two-lane rural roads," IET Intelligent Transport Systems, vol. 7, no. 2, pp. 182-189, 2013.

[16] X. Zeng and J. Wang, "A stochastic driver pedal behavior model incorporating road information," IEEE Transactions on Human-Machine Systems, vol. 47, no. 5, pp. 614-624, 2017.

[17] Liqun, C. Peng, W. Zhen, and M. Huang, "Novel vehicle motion model considering driver behavior for trajectory prediction and driving risk detection," Transportation Research Record Journal of the Transportation Research Board, vol. 2434, no. 1, pp. 123-134, 2014.

[18] H. Cai, Z. Hu, Z. Chen, and D. Zhu, "A driving fingerprint map method of driving characteristic representation for driver identification," IEEE Access, vol. 6, pp. 71 012-71 019, 2018.

[19] C. Miyajima and K. Takeda, "Driver-behavior modeling using on-road driving data: a new application for behavior signal processing," IEEE Signal Processing Magazine, vol. 33, no. 6, pp. 14-21, 2016.

[20] P. Angkititrakul, C. Miyajima, and K. Takeda, "Modeling and adaptation of stochastic driver-behavior model with application to car following," in Proceeings of the 2011 IEEE Intelligent Vehicles Symposium (IV), pp. 814-819, Baden-Baden, Germany, June 2011.

[21] B. Shi, X. Li, H. Jie, T. Yun, and L. Hui, "Evaluating driving styles by normalizing driving behavior based on personalized driver modeling," IEEE Transactions on Systems Man \& Cybernetics Systems, vol. 45, no. 12, pp. 1502-1508, 2015.

[22] S. Yamada and M. Takahashi, "Analysis of driver behavior based on real expressway data," IFAC-PapersOnLine, vol. 49, no. 19 , pp. 337-342, 2016.

[23] T. Taniguchi, S. Nagasaka, K. Hitomi, K. Takenaka, and T. Bando, "Unsupervised hierarchical modeling of driving behavior and prediction of contextual changing points," IEEE Transactions on Intelligent Transportation Systems, vol. 16, no. 4, pp. 1746-1760, 2015.

[24] H. Okuda, N. Ikami, T. Suzuki, Y. Tazaki, and K. Takeda, "Modeling and analysis of driving behavior based on a probability-weighted arx model," IEEE Transactions on Intelligent Transportation Systems, vol. 14, no. 1, pp. 98-112, 2013.

[25] D. Shi, L. Chu, J. Guo, G. Tian, Y. Feng, and Z. Li, "Energy control strategy of heb based on the instantaneous optimization algorithm," IEEE Access, vol. 5, pp. 19 876-19 888, 2017.

[26] L. Zhang, L. Zhang, S. Liu, J. Zhou, and C. Papavassiliou, "“Three-dimensional underwater path planning based on modified wolf pack algorithm," IEEE Access, vol. 5, pp. 22783-22 795, 2017.

[27] B. B. Munyazikwiye, H. R. Karimi, and K. G. Robbersmyr, "Optimization of vehicle-to-vehicle frontal crash model based on measured data using genetic algorithm," IEEE Access, vol. 5, pp. 3131-3138, 2017.

[28] S. B. Amsalu and A. Homaifar, "Driver behavior modeling near intersections using hidden markov model based on genetic algorithm," in Proceedings of the 2016 IEEE International Conference on Intelligent Transportation Engineering (ICITE), pp. 193-200, Singapore, Singapore, August 2016.
[29] J. Liu, L. Feng, and Z. Li, “The optimal road grade design for minimizing ground vehicle energy consumption," Energies, vol. 10, no. 5, p. 700, 2017.

[30] Caltrans Performance Measurement System (Pems),"http:// pems.dot.ca.gov/, California Department of Transportation.

[31] G. E. Hinton, S. Osindero, and Y.-W. Teh, "A fast learning algorithm for deep belief nets,” Neural Computation, vol. 18, no. 7, pp. 1527-1554, 2006.

[32] Y. Bengio, Learning Deep Architectures for AI, Piscataway, NJ, USA, 2009, https://ieeexplore.ieee.org/document/8187120.

[33] S. Mirjalili and A. Lewis, "The whale optimization algorithm," Advances in Engineering Software, vol. 95, pp. 51-67, 2016.

[34] M. Abdel-Basset, M. Gunasekaran, D. El-Shahat, and S. Mirjalili, "A hybrid whale optimization algorithm based on local search strategy for the permutation flow shop scheduling problem," Future Generation Computer Systems, vol. 85, 2018.

[35] H. M. Mohammed, S. U. Umar, and T. A. Rashid, "A systematic and meta-analysis survey of whale optimization algorithm," Computational Intelligence and Neuroscience, vol. 2019, p. 25, Article ID 8718571, 2019.

[36] K. Inata, P. Raksincharoensak, and M. Nagai, "Driver behavior modeling based on database of personal mobility driving in Urban Area," in Proceedings of the 2008 International Conference on Control, Automation and Systems, pp. 2902-2907, Seoul, South Korea, October 2008. 